\title{
Simulation and optimization for separation of ethylenediamine-water azeotropic mixture
}

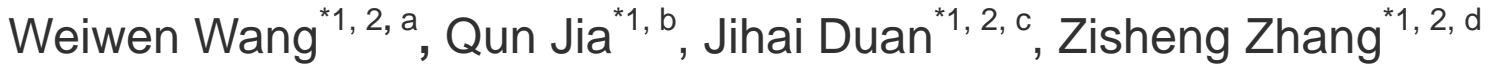 \\ ${ }^{1}$ College of Chemical Engineering, Qingdao University of Science and Technology, Qingdao, P.R. \\ China.266042 \\ ${ }^{2}$ Ecological Chemical Base \\ awwwang@qust.edu.cn, banqunaita@sina.com, 'duanjihai@yahoo.com.cn, ${ }^{\mathrm{d}}$ zhangzz77@yahoo.co \\ $\mathrm{m}$
}

Keywords: Aspen Plus, ethylenediamine, azeotro- pic distillation

\begin{abstract}
In this paper, the separation process of ethylenediamine-water azeotropic mixture is investigated with Aspen Plus software. The sim- ulation results show that the extractive disti- llation column includes 26 theoretical plates, the D/F of azeotropic distillation column is 0.18 , the feed location of azeotropic distillation column is 22nd theoretical plate and the azeotropic agent velocity is $200 \mathrm{kmol} / \mathrm{h}$. The purity of the produc- $\mathrm{t}$ is $99 \mathrm{wt} \%$ of ethylenediamine.
\end{abstract}

\section{Introduction}

Ethylenediamine is a kind of intermediates of various functional products. It is used in the pharmaceutical production, pesticide, epoxy curing agent, fuel intermediates, dye fixing agent and EDTA. Ethylenediamine can prod- uce chelating agent, pest control agent, lubric- ant, rubber accelerator [1-3]. Dichloroethane, ethylene glycol and monoethanolamine can be used to produce ethylenediamine. There are several advantages of ethylene glycol as the raw materials, such as low cost and the simple reaction steps. However, under ordinary pressure, ethylenediamine and water can form an azeotropic mixture, the temperature of mixture is $119.5{ }^{\circ} \mathrm{C}$, and the composition of ethylenediamine is $81.6 \mathrm{wt} \%$, so they are not easily separated[4-8].

The ethyleneiamine-water T-x-y diagram is shown below by character analysis of the Aspen Plus software (Fig.1).

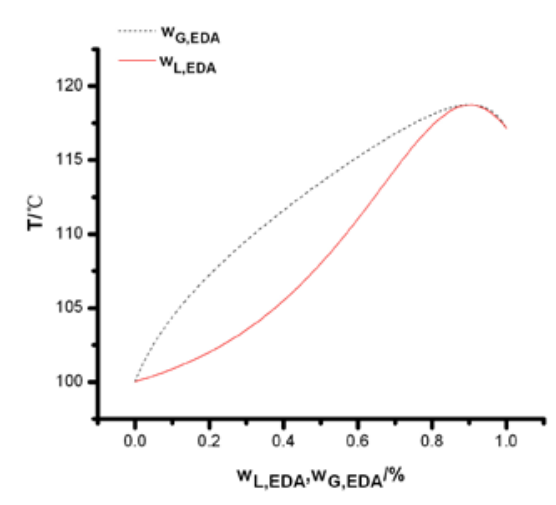

Figure 1.T-wL- wG diagram for Ethylenediamine/water

WG is the vapor mass fraction and $w \mathrm{~L}$ is the liquid mass fraction. Obviously we can find t- he azeotropic point, so the conventional meth- od is very difficult to separate this system.

\section{Azeotropic Process}

Tian Zhongshe[9] from Northwestern Univ- ersity used toluene and benzene as azeotropic agent to remove ethylene diamine, and the piperazine concentration of the product was $97 \mathrm{wt} \%$. 
The advantage of benzene as the entrainer has two points, one is lower cost than other azeotropic agent and the other is that benzene is a normal chemical[10-14].

The processed products enter into the bottom of azeotropic distillation column. The top products of column (water and most of the entrainer) are separated in the phase separator, and the bottom products of column enter into the separation column to separate. The entrainer flows into the mixer. Process is shown in figure 2.

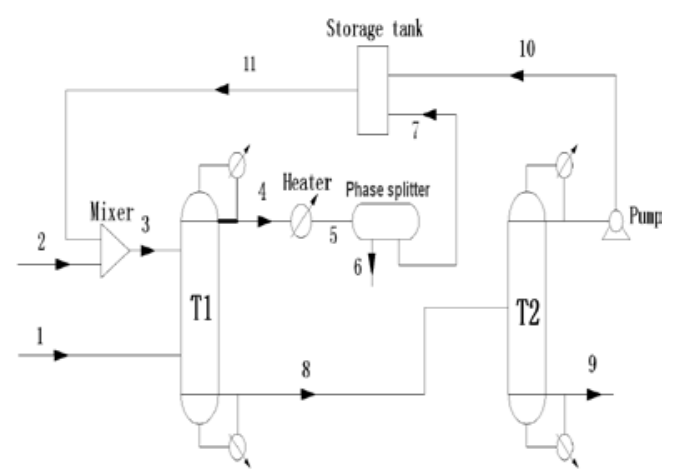

T1- Azeotropic distillation column T2- Azeotropic separation column

Figure 2.Azeotropic process flow diagram of ethylenediamine

RADFRAC module is used as Rectification model, and the thermodynamic model is adopt- ed to depict the UNIFAC equation. There are several impact factors on the separation process such as the amount of entrainer, feed temperature, feed location, number of stages and the reflux ratio. The optimal conditions are obtained by the sensitivity analysis.

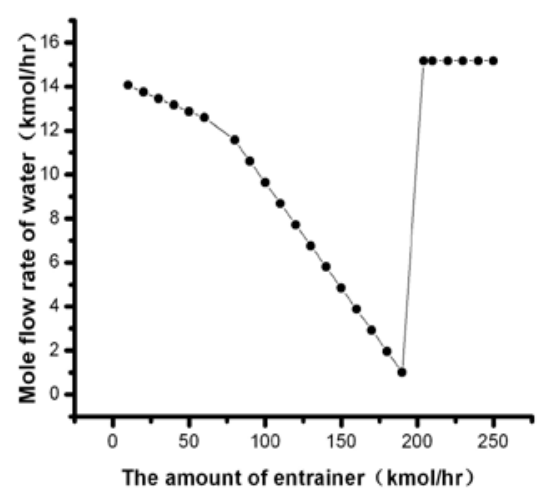

Figure3.Sensitivity analysis of the amount of entrainer

Figure 3 revealed that the amount of ethyl- enediamine is decreased with the addition of benzene. When the amount of the entrainer was added to $200 \mathrm{kmol} / \mathrm{h}$, the water of the product was basically completely separated.

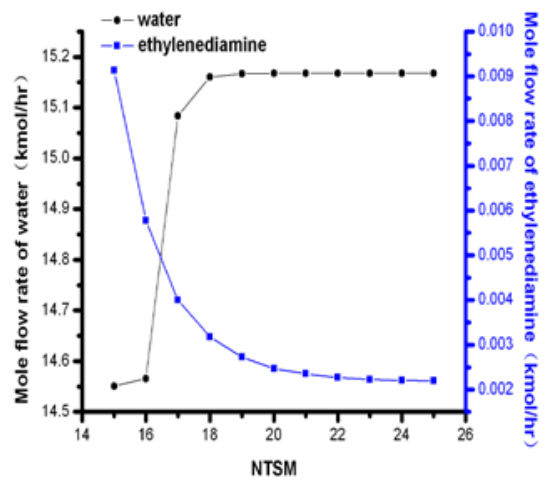

Figure 4.Sensitivity analysis of NTSM

It can be seen from the figure 4 that when achieving 20 stages of column, the mole flow rate of water is close to $15.1 \mathrm{kmol} / \mathrm{h}$, whil- e mole flow rate of ethylenediamine is less than or equal to $0.01 \mathrm{kmol} / \mathrm{hr}$. Due to the restriction of the cost and actual operation, the number of plates should be 
26.

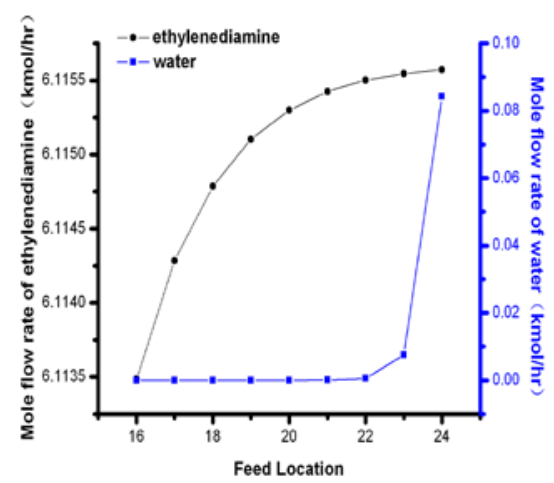

Figure 5.Sensitivity analysis of feed stage

It can be seen from Figure 5, with the feed location near the tower kettle, the mole flow rate of ethylenediamine is increasing, but there were a little of water in the bottom products. The feed location of azeotropic column should be 22nd plate.

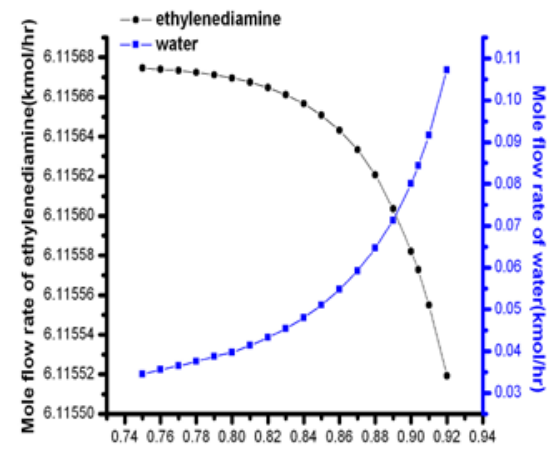

DiF

Figure 6.Sensitivity analysis of D/F
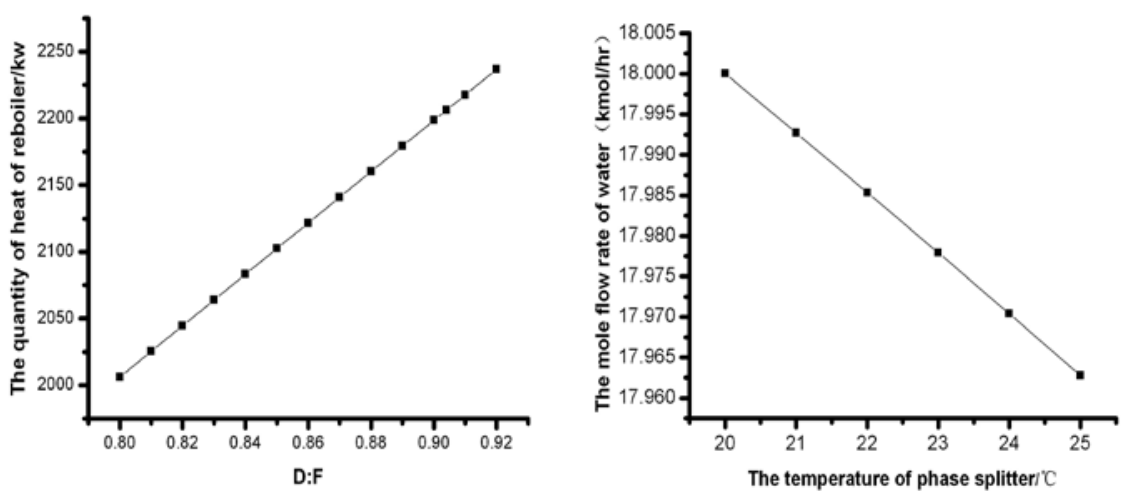

Figure 7.Sensitivity analysis of quantity of heat Figure 8.Sensitivity analysis of the temperature of phase splitter

Figure 6 shows that the mole flow rate of ethylenediamine decrease in a very small range, and the mole flow rate of water increase slowly. Data presented in figure 7 reveal that quantity of heat increase with the increase of $\mathrm{D} / \mathrm{F}$. The $\mathrm{D} / \mathrm{F}$ of azeotropic column is 0.8 .

As is shown in the picture, with the increase of temperature of phase splitter, the mole flow rate of water decreased. Water and azeotroping agent could be well separated at $20^{\circ} \mathrm{C}$.

\section{Conclusions}

Azeotropic distillation is used to separate ethylenediamine -water system and azeotropi- 
c agent can be recycled to azeotropic column. The purity of ethylenediamine is $99 \mathrm{wt} \%$. Th- rough the optimization of azeotropic distil- lation process, the parameters of azeotropic distillation are obtained. The azeotropic distillation column includes 26 theoretical plates, the feed stage of azeotropic distillation column is $22 \mathrm{nd}$ plate, the $\mathrm{D} / \mathrm{F}$ of azeotropic distillation column is 0.18 , azeotropic agent v- elocity is $200 \mathrm{kmol} / \mathrm{h}$.

\section{Acknowledgment}

This work was supported by the National Natural Science Foundation (21276132) and the Livelihood Project Foundation of Qingdao (13-1-3-128-nsh).

\section{References}

[1] Yang Yi, Tian Baoliang. Process in research of ethylenediamine synthesis. Petrochemical Technology. vol. 41, pp. 603-607, (2012)

[2] Zhao Fengwei, Yang Jiaming. Study on synthesis of ethylenediamine over modified mordenite catalyst. Industrial Catalysis. vol. 16, pp. 70-72, (2008)

[3] He Taoji. Simulation and experiment for separation of acetonitrile - water azeotropic mixture. Tianjin University, Jun. (2008).

[4] Yang Jianming, Lv Jian, Wang Wei. Method for purification of ethylenediamine. 101723837. Jun. (2010).

[5] He Miaoli, Liu Gong, Yang Bolun. Simulation of distillation based on Aspen plus for products from catalytic amination of ethanolamine. Modern Chemical Industry. vol. 2, pp. 24-27, (1993)

[6] Wang Yanming, Yao Xiaolong. Research advances in synthesis and separation of ethanoldiamine. Guangzhou Chemical Industry. vol.38, pp, 49-52, (2010)

[7] Kong Wangqing, Zhang Liangxiao, Xie Jingsi. Application of ethylenediamine and development of downstream products. Hebei Chemical Indust- ry.vol.3, pp. 13-15, (2005)

[8] Zong Hongyuan. Production Technology and Market of Ethylenediamine. Advances in Fine Petrochemicals. vol.3, pp. 49-51, 2002

[9] Tian Zhongshe, Liang Jianguo. Separation and simulation of products from directcyclization of ethylenediamine. Chemical Engineering. vol.40, pp. 67-70, (2012)

[10]Zhang Peixun. Research on the purification of ethylenediamine. Shanghai Chemical Industry. vol.10, pp. 16-20, (1996)

[11]Air Products and Chemicals Inc. Production of ethylenediamine from monoethanolamine and ammonia: US, 4918233. 1990 - $04-17$.

[12]Texaco Chemical Inc. Decolorization of Polyethylene Polyamines Using Ruthenium: US, 5364971. (1994).

[13]Han Changing, Song Xiaoping. Study on the synthesis of ethylenediamine with a tubular reactor. Zhejiang University, Jun. (2008)

[14]Huang Kelin, Wang Zefen. Research progress of ethylenediamine synthesis. Technology \& Development of Chemical Industry. vol. 8, (2010) 